\title{
UNIVERSITYOF
}

FORWARD

THINKING

WESTMINSTER用

WestminsterResearch

http://www.westminster.ac.uk/westminsterresearch

\section{Simulating the Effects of Skin Thickness and Fingerprints to Highlight Problems with Non-invasive RF Blood Glucose Sensing from Fingertips}

Turgul, V. and Kale, I.

This is a copy of the author's accepted version of a paper subsequently published in the IEEE Sensors Journal, 17 (22), pp. 7553-7560.

It is available online at:

https://dx.doi.org/10.1109/JSEN.2017.2757083

(c) 2017 IEEE . Personal use of this material is permitted. Permission from IEEE must be obtained for all other uses, in any current or future media, including reprinting/republishing this material for advertising or promotional purposes, creating new collective works, for resale or redistribution to servers or lists, or reuse of any copyrighted component of this work in other works.

The WestminsterResearch online digital archive at the University of Westminster aims to make the research output of the University available to a wider audience. Copyright and Moral Rights remain with the authors and/or copyright owners.

Whilst further distribution of specific materials from within this archive is forbidden, you may freely distribute the URL of WestminsterResearch: ((http://westminsterresearch.wmin.ac.uk/)).

In case of abuse or copyright appearing without permission e-mail repository@westminster.ac.uk 


\title{
Simulating the Effects of Skin Thickness and Fingerprints to Highlight Problems with Non-invasive RF Blood Glucose Sensing from Fingertips
}

\author{
Volkan Turgul, Student Member, IEEE and Izzet Kale, Member, IEEE
}

\begin{abstract}
The non-invasive measurement of blood glucose is a popular research topic where $\mathrm{RF} / \mathrm{microwave}$ sensing of glucose is one of the promising methods in this area. From the many available measurement sites in the human body, fingertips appear to be a good choice due to a good amount of fresh blood supply and homogeneity in terms of biological layers present. The non-invasive RF measurement of blood glucose relies on the detection of the change in the permittivity of the blood using a resonator as a sensor. However, the change in the permittivity of blood due to the variation in glucose content has a limited range resulting in a very small shift in the sensor's frequency response. Any inconsistency between measurements may hinder the measurement results. These inconsistencies mostly arise from the varied thickness of the biological layers and variation of fingerprints that are unique to every human. Therefore, the effects of biological layers and fingerprints in fingertips were studied in detail and are reported in this paper.
\end{abstract}

Index Terms — Glucose; Non-invasive; Permittivity; RF

\section{INTRODUCTION}

$\mathrm{D}$ IABETES Mellitus (DM) is a disease associated with high Blood Glucose Levels (BGL) affecting over 400 million people worldwide and this number increases rapidly [1]. The normal BGL ranges from $72 \mathrm{mg} / \mathrm{dl}$ to $108 \mathrm{mg} / \mathrm{dl}$ whereas patients with DM may experience levels as high as $400 \mathrm{mg} / \mathrm{dl}$. In this case, the BGL is advised to be regulated through medication or insulin shots to reduce the chance of long-term negative health effects [2]. The conventional measurement methods are invasive, where an accurate BGL measurement can be taken at health clinics/hospitals. However, mostly portable BGL measurement devices are preferred by patients for convenience that allow for an instant reading, anytime and anywhere. In this method, a small blood sample is obtained from the fingertip, which is then applied onto a test strip.

*Research supported by University of Westminster Scholarship.

The authors are with the Applied DSP and VLSI Research Group (ADVRG), Department of Engineering, Faculty of Science and Technology, University of Westminster, W1W 6UW, London, U.K. (e-mail: volkan.turgul@my.westminster.ac.uk; kalei@westminster.ac.uk).
The test strip is inserted into the measurement device for a BGL reading. Depending on the severity of the disease, a patient may need to take up to 10 measurements a day, which causes discomfort. Furthermore, the blood contaminated consumables such as test strips and lancets should be disposed of properly to avoid the spread of blood-related diseases. Therefore, a non-invasive BGL measurement device is highly desirable to avoid the discomfort and the aforementioned risks. Researchers have been working on various non-invasive BGL measurement methods to measure BGL non-invasively [3-5]. Among these methods, the optical method can be considered as a notable one due to its potential to solve the problem using specific optical wavelengths where glucose shows strong absorption [6]. Another exciting yet less relevant method to the non-invasive methods is the minimally-invasive measurement systems using micro-needles [7] or implantable sensors [8]. Having access to the measurement media such as interstitial fluid or blood, these systems face less challenge in terms of glucose measurement. However, prolonging sensor life in the body by utilizing bio-compatible materials remains a challenge for minimally-invasive systems.

One other popular and promising non-invasive method is the Radio Frequency (RF)/microwave sensing. There are a number publications reporting on dielectric modeling and measurement of glucose, non-invasive sensor design such as [9-15], as well as few companies developing commercial noninvasive RF BGL measurement devices [16, 17]. An RF sensor is used to measure the change in the dielectric properties of blood resulting from the variation of BGL[1819]. This change causes a frequency shift in the sensor's frequency response. However, detecting this change is challenging as the expected frequency shift is less than $8 \mathrm{MHz}$ for the realistic BGL range and error sources such as the make-up of the fingertip, its positioning and the pressure applied onto the sensor dominate the shift in the frequency response [20-22]. Hence, these factors were studied in detail to observe their effects and propose novel solutions. 


\section{Non-INVASIVE RF Glucose SENSOR AND MEASUREMENT}

The estimation of blood glucose is done by placing a body part (sample) onto a sensor as shown in Fig. 1 and observing its frequency response.

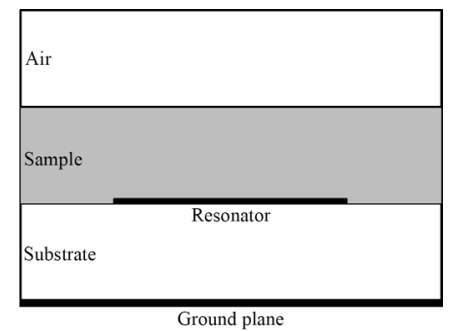

Fig. 1. Cross-section of the sensor and the sample

A compact and sensitive non-invasive RF glucose sensor that was previously designed and reported in [21] was used in this study to perform the simulations and measurements. The sensor is a single-port resonator on a 32 mil thick RO3006 substrate with an unloaded resonance frequency of $4.8 \mathrm{GHz}$ where the resonance shifts down to about $3.25 \mathrm{GHz}$ when a fingertip is placed on it. The layout of the sensor used is shown in Fig. 2.

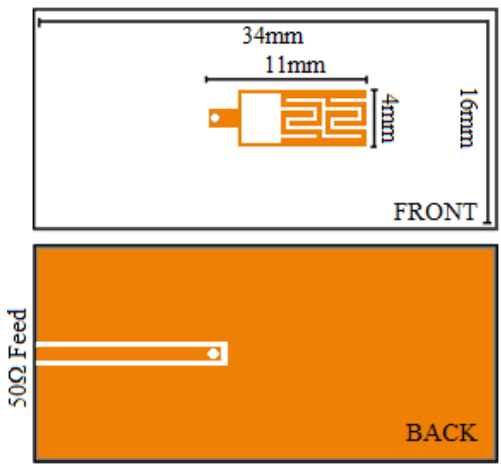

Fig. 2. Layout of the sensor used

The sensor was simulated in CST Microwave Studio (MWS) [23] and measured using a Rohde \& Schwarz ZVL6 Vector Network Analyzer (VNA). The simulated and measured responses are shown in Fig. 3.

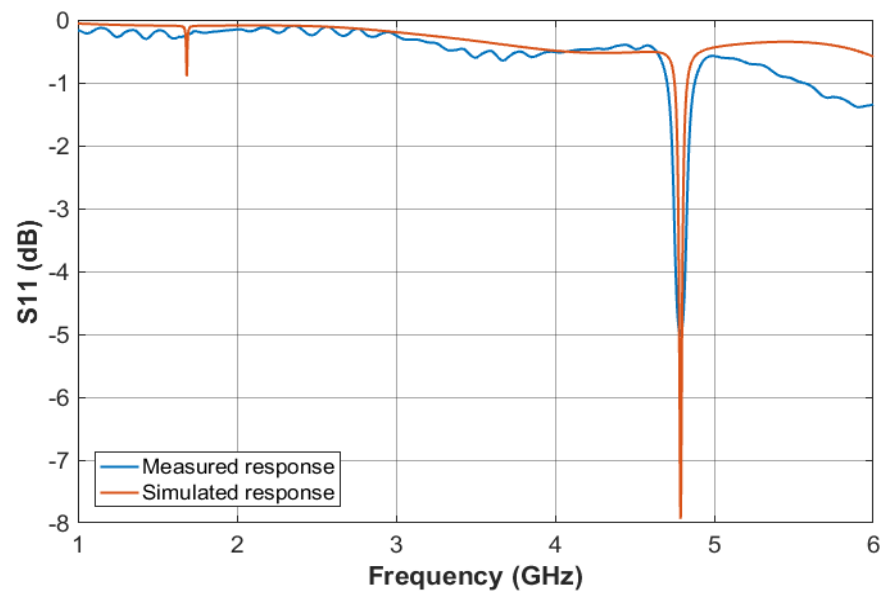

Fig. 3. Simulated and measured responses of the sensor
Deionized water and glucose phantoms comprising deionized water and pure D-glucose with concentrations of $100 \mathrm{mg} / \mathrm{dl}$, $250 \mathrm{mg} / \mathrm{dl}, 500 \mathrm{mg} / \mathrm{dl}$ and $1000 \mathrm{mg} / \mathrm{dl}$ were measured with the sensor. The samples were prepared this way to solely observe the effects of glucose; hence, no other constituents were added to make the fluid more physiological-relevant. A Perspex plate was placed on the sensor with a circular opening over the active region of the sensor holding $125 \mu \mathrm{l}$ sample volume. Each sample was measured 10 times and the results were averaged. The measurement results are shown in Fig. 4.

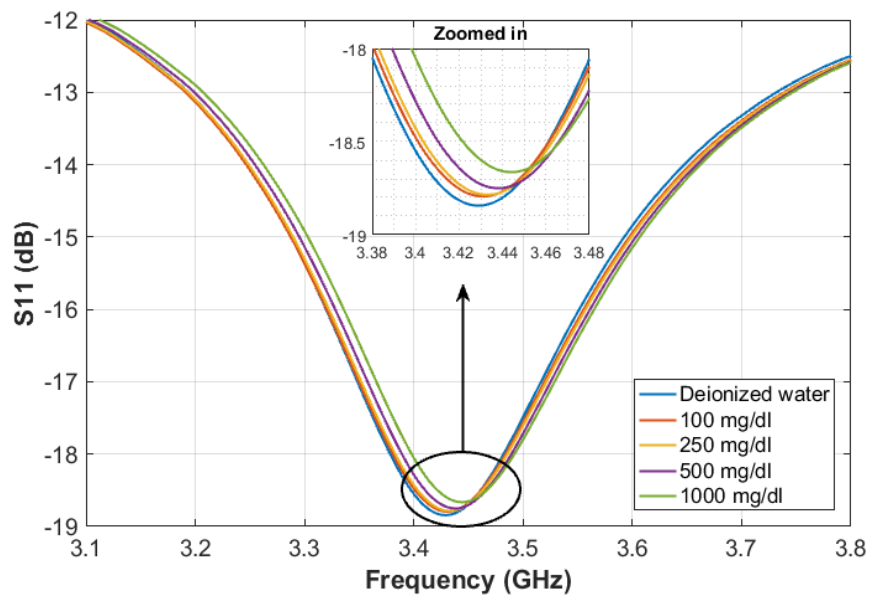

Fig. 4. Measured response of the sensor with glucose phantoms

For a better understanding of the relationship between glucose concentration and resonance frequency, the resonance frequency value for each concentration was normalized to the resonance frequency observed with deionized water. The results are shown in Fig. 5 along with confidence intervals for 10 measurements.

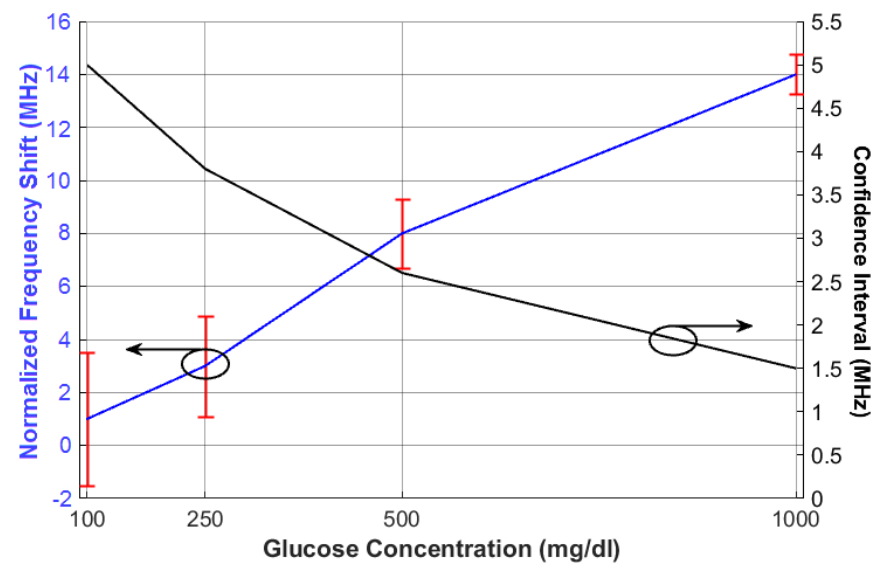

Fig. 5. Normalized frequency shift for various glucose concentrations and confidence interval

As it can be seen, the confidence interval is wider for low glucose concentrations making it harder to achieve accurate and repeatable measurements. A frequency shift of $14 \mathrm{MHz}$ was observed between $0 \mathrm{mg} / \mathrm{dl}$ and $1000 \mathrm{mg} / \mathrm{dl}$ solutions and the shift is almost linear, which translates to a frequency

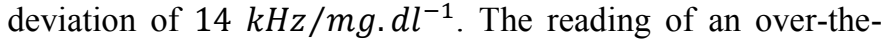
counter BGL meter must produce results within $\pm 15 \%$ of the 
real BGL value set by the International Standardization Organization (ISO) regulations [24]. For example, for a concentration of $100 \mathrm{mg} / \mathrm{dl}$ the acceptable range is $85-115$ $\mathrm{mg} / \mathrm{dl}$, which means that the RF measurement accuracy must be greater than or equal to $420 \mathrm{kHz}$ in an ideal situation. However, it can be seen in Fig. 5 that the measurement accuracy is within $5 \mathrm{MHz}$ instead.

It is even harder to take accurate measurements from body parts as human tissues are lossy and there are many constituents in blood beside glucose contributing to the measurement results. For the realistic BGL range the expected shift is less than $8 \mathrm{MHz}$. Moreover, there are several error mechanisms in taking measurements that may introduce a shift over $100 \mathrm{MHz}$, which are explained in the following sections. For this reason, any source of error in the measurement must be eliminated or greatly reduced in order to get an acceptably accurate measurement.

\section{NON-INVASIVE Glucose Measurement From the FINGERTIPS}

The permittivity of blood changes as the concentration of glucose level varies. The fingertip is a suitable measurement site but there are a number of things that play a fundamental and pivotal role in the measurement accuracy and repeatability. These are; the fingerprints, skin thickness, the pressure applied by the fingertip while taking a measurement and finger positioning on the sensor.

The fingertips are imprinted with fingerprints, which are uniquely patterned in each finger and each person. They are formed of ridges and valleys with an average depth of $50 \mu \mathrm{m}$ [25]. Although this appears to be a small feature to be neglected in non-invasive BGL measurements or simulations, the frequency shift caused by the fingerprints is greater than that caused by the actual glucose level variation. This is because the valleys introduce air gaps whose permittivity is much lower than skin's permittivity and this lowers the effective permittivity seen by the RF sensor. This is illustrated in Fig. 6.

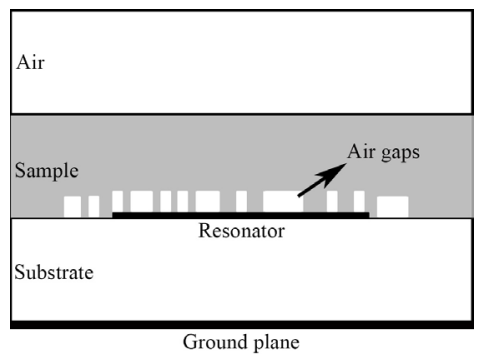

Fig. 6. Cross-section of the sensor and the sample with air gaps.

The frequency response is affected differently depending on the depth of the valleys and finger positioning on the sensor due to the irregular shape of the fingerprints. Hence, the effects of valley depth and fingerprint pattern were investigated.

The finger can be represented by a 4-layer equivalent model as shown in Fig. 7 [19].

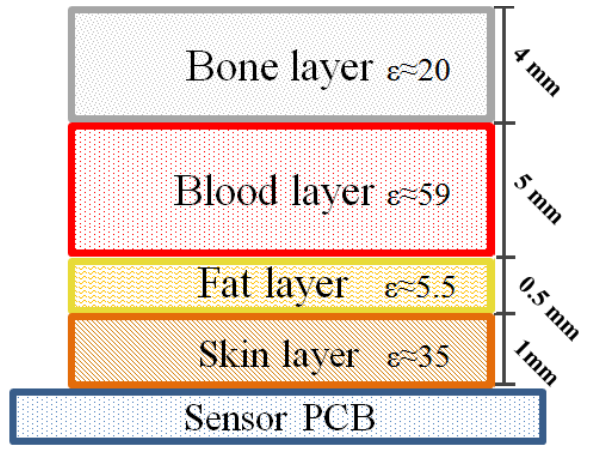

Fig. 7. 4-layer model of fingertip.

Skin is the first biological layer on the sensor; therefore, it has a pronounced effect on the frequency response of the sensor. Any variation in the thickness of this layer directly affects the response. The ridges in the fingerprints are compressed as pressure is applied onto the sensor by the finger. This alters the depth of the valleys, hence the volume of the air gaps and the skin thickness, which plays a role on the frequency response. Moreover, the fat and blood layers are also compressed depending on the pressure applied and the blood is pushed away from the fingertip if too much pressure is applied. The effects of these issues were studied and are reported in the following sections.

\section{EFFECTS OF FINGERPRINTS}

The effects of fingerprints in non-invasive measurements were first reported in [22]. However, only the effect of presence of a fingerprint was investigated and it was shown that it causes a frequency shift in the sensor's frequency response. This work was expanded by investigating the effects of different fingerprint patterns with varied valley depths. For this, five random fingerprints were obtained from the internet and their patterns were randomly altered to ensure anonymity. The patterns were imprinted on the skin layer of the 4-layer fingertip model in CST MWS as shown in Fig. 8. The edges of the fingerprints were left rectangular instead of rounded to simplify the 3D modeling. However, this is expected to have little to no effect on simulation results. The model measures $25 \mathrm{~mm} \times 18 \mathrm{~mm}$ in an oval shape to resemble an average human index finger. Simulations were performed by varying the valley depth in each fingerprint with values of $5 \mu \mathrm{m}, 10$ $\mu \mathrm{m}, 20 \mu \mathrm{m}, 40 \mu \mathrm{m}$ and $80 \mu \mathrm{m}$, assuming a $50 \mu \mathrm{m}$ average valley depth for an uncompressed fingerprint [25, 26]. Mesh size was set to 20 cells at $5 \mathrm{GHz}$ in MWS.

TABLE I

VALLEY DEPTH VS RESONANCE FREQUENCY (MHZ)

\begin{tabular}{cccccc}
\hline \hline Depth & FP* 1 & FP 2 & FP 3 & FP 4 & FP 5 \\
\hline $0 \mu \mathrm{m}$ & 3228 & 3228 & 3228 & 3228 & 3228 \\
$5 \mu \mathrm{m}$ & 3236 & 3233 & 3234 & 3238 & 3236 \\
$10 \mu \mathrm{m}$ & 3238 & 3237 & 3235 & 3239 & 3240 \\
$20 \mu \mathrm{m}$ & 3242 & 3238 & 3235 & 3244 & 3241 \\
$40 \mu \mathrm{m}$ & 3284 & 3240 & 3241 & 3240 & 3246 \\
$80 \mu \mathrm{m}$ & 3328 & 3262 & 3282 & 3324 & 3356 \\
$*$ FP (Fingerprint) & & & &
\end{tabular}




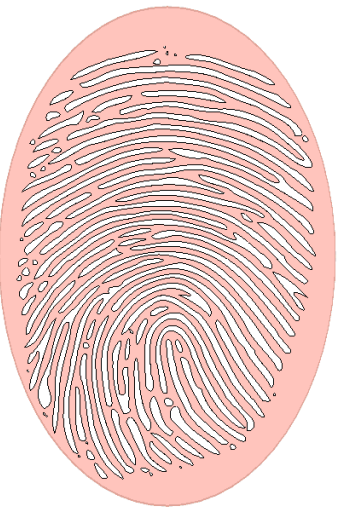

a)

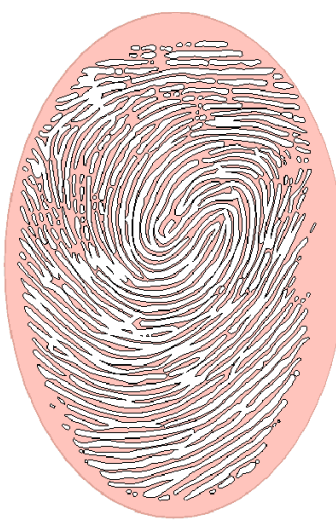

c)

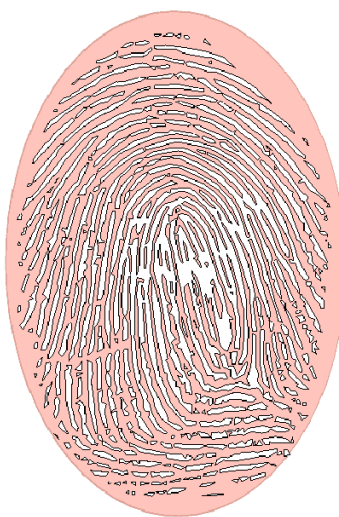

e)

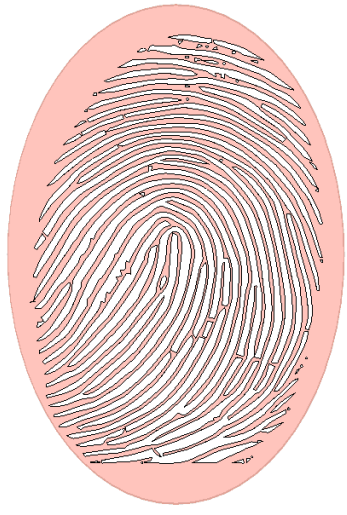

b)

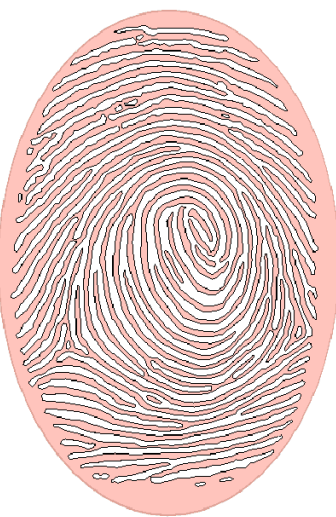

d)

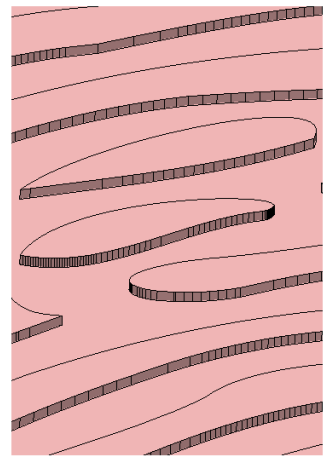

f)
Fig. 8. Fingerprint a) pattern $1 \mathrm{~b}$ ) pattern $2 \mathrm{c}$ ) pattern $3 \mathrm{~d}$ ) pattern 4 e) pattern $5 \mathrm{f}$ ) close-up section of the $3 \mathrm{D}$ ridges and valleys in CST MWS

The main resonance point of the sensor for each fingerprint pattern and valley depth value was recorded and are given in Table I. As it can be seen, the resonance point is different for every pattern and it changes with the varying valley depth values. As the depth of the valleys increases the resonance frequency increases because the effective permittivity seen by the sensor decreases. The valley depth varies from person to person, however, it also varies by the amount of pressure applied onto the sensor by the fingertip due to compression. For this reason, attention must be paid to monitoring the pressure applied, otherwise, the sensor response will differ in each measurement.
The relationship between the valley depth and frequency offset for each pattern in shown in Fig. 9.

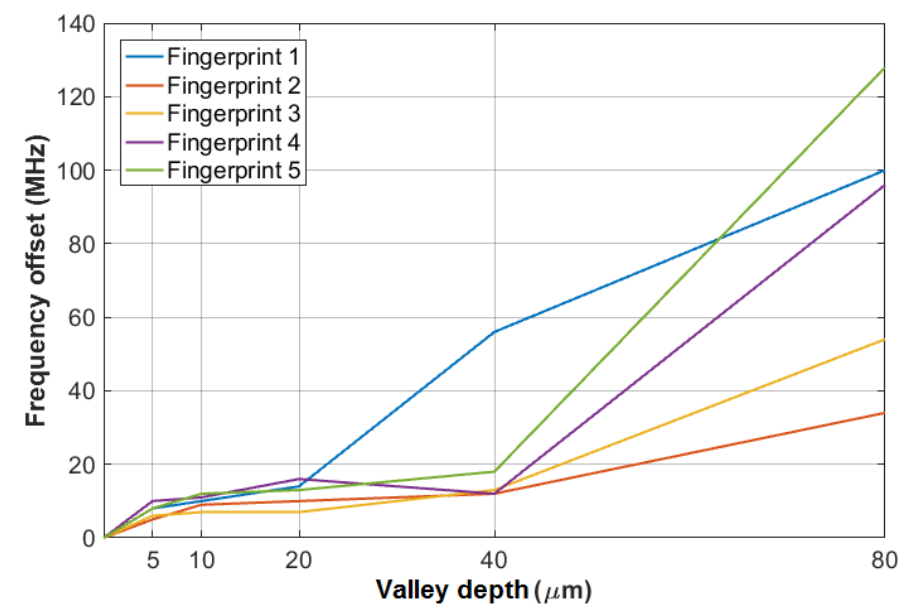

Fig. 9. Frequency offset vs. valley depth for different fingerprint patterns

Simulation results show that the frequency shift due to valley depth and fingerprint variations may be as high as $100 \mathrm{MHz}$ in each case. This marks the importance of the need to be aware of and take account of the fingerprints in noninvasive BGL measurements.

Moreover, as reported in [22], fingertip positioning is also a very important factor to pay attention to while taking measurements as a positioning error may cause a frequency shift well over $100 \mathrm{MHz}$. Due to the asymmetrical nature of fingerprints, the error caused differs in magnitude depending on where the finger is positioned on the sensor, impacting the repeatability of the measurements. This was shown by performing a simulation where the fingertip's position was varied between $-2 \leq x \leq 2$ and $-2 \leq y \leq 2$, and the resulting resonance frequency for each point was normalized with respect to the frequency at the center, $(x, y)=(0,0)$, where $x$ and $y$ are coordinate points in millimeters. The resulting graph is shown in Fig. 10.

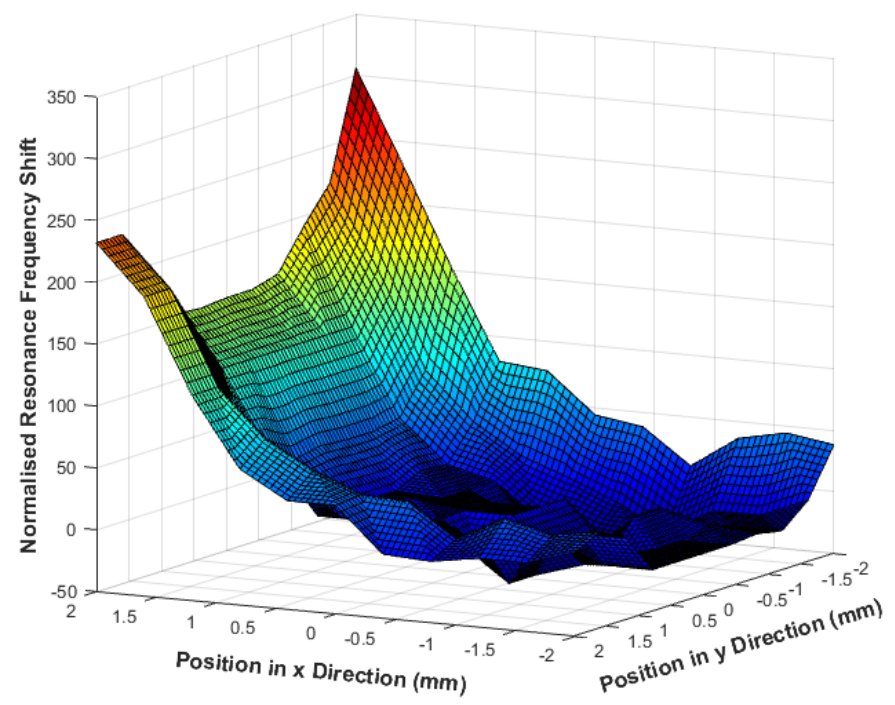

Fig. 10. Normalized frequency shift vs. fingertip position on the sensor 
Furthermore, it should be noted that when a fingertip is used for measurement, any contaminants such as oil, sweat and dirt between the fingertip and the sensor would adversely affect the measurement accuracy. The severity of the effect depends on the quantity, distribution and dielectric properties of the contaminant. We performed a set of experiments on several volunteers where we affixed the sensor onto the participants' index finger and asked them to stay still for 10 minutes. We observed for each participant that the resonance frequency decreased non-linearly during the span of the measurement as their fingertips started sweating. The sweating introduced up to an $80 \mathrm{MHz}$ shift in the frequency response, depending on the sweating rate and pattern of each participant. This was expected as the dielectric constant is about double that of skin.

\section{EFFECT OF Biological LAYER THICKNESS}

Next, the effects of skin, fat and blood layers' thickness were studied. The thickness of the skin layer was varied from $0.25 \mathrm{~mm}$ to $1 \mathrm{~mm}$ to observe its effect on the resonance frequency. The skin is not compressed much with the pressure applied onto the sensor, however, its thickness varies from person to person. The thickness of the skin clearly affects the resonance frequency of the sensor as shown in Fig. 11. A $0.25 \mathrm{~mm}$ difference in the thickness introduces about a 100 $\mathrm{MHz}$ shift.

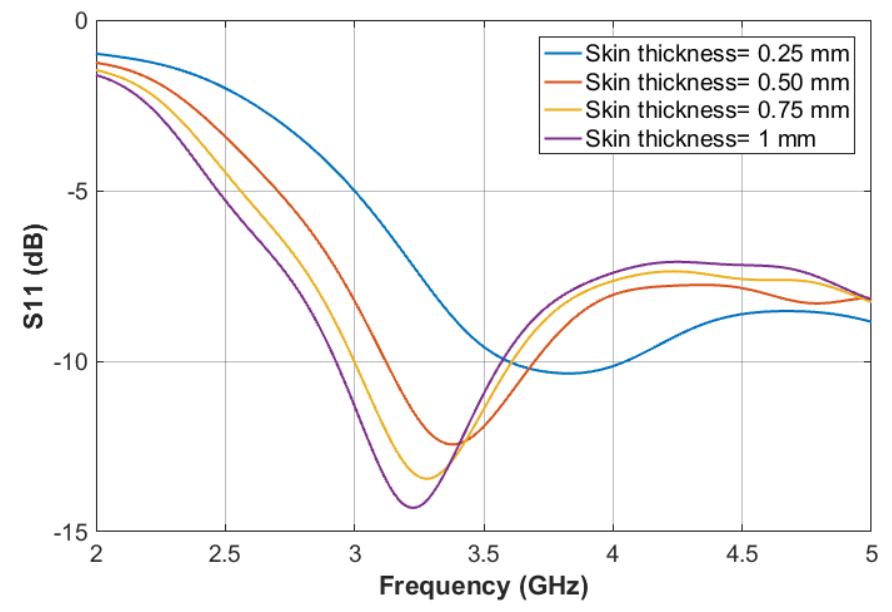

Fig. 11. Resonance frequency for various skin thickness values

The overall thickness of the fingertip reduces with the pressure applied because the tissues are compressed. In the original 4-layer model it is assumed that the layers are ordered and aligned. However, in reality blood exists in a complex network of capillaries and soft tissue. Therefore, varying the thicknesses of fat and blood layers alone does not wholly reflect reality. For this reason, a new voxelized model was investigated and is proposed. The cross-sections of the original and voxelized models in CST MWS are shown in Fig. 12. The fat and blood layers were voxelized with a volume of $0.4 \mathrm{~mm} \times 0.4 \mathrm{~mm} \times 0.4 \mathrm{~mm}$ (8880 total voxels).

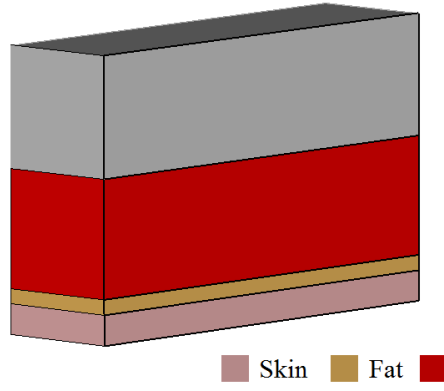

a)

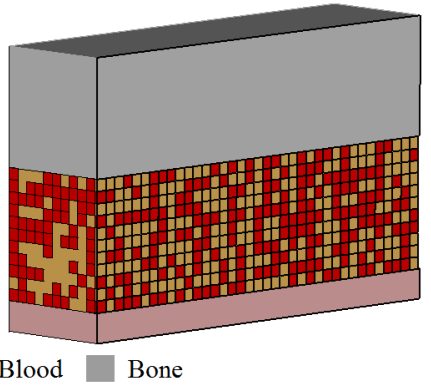

b)
Fig. 12. 4-layer fingertip model in CST MWS a) original b) voxelized

This voxel size was selected as a trade-off between model resolution and voxel generation/simulation time. The blood and fat voxels were randomly generated and distributed with $50 \%$ probability between the skin and bone layers. The model was simulated and its response was compared with the original model's response as well as the measured response of the sensor with an actual human fingertip. The results are shown in Fig. 13. The model was simulated 5 times by regenerating the voxels randomly to observe the effect of distribution of the voxels on the frequency response of the sensor. A standard deviation of $230 \mathrm{kHz}$ was observed between simulations which shows that the random voxel generation offers good repeatability. The blood voxels can be replaced with glucose/water Cole-Cole permittivity relaxation model [20] to investigate the effect of glucose concentration on the frequency response.

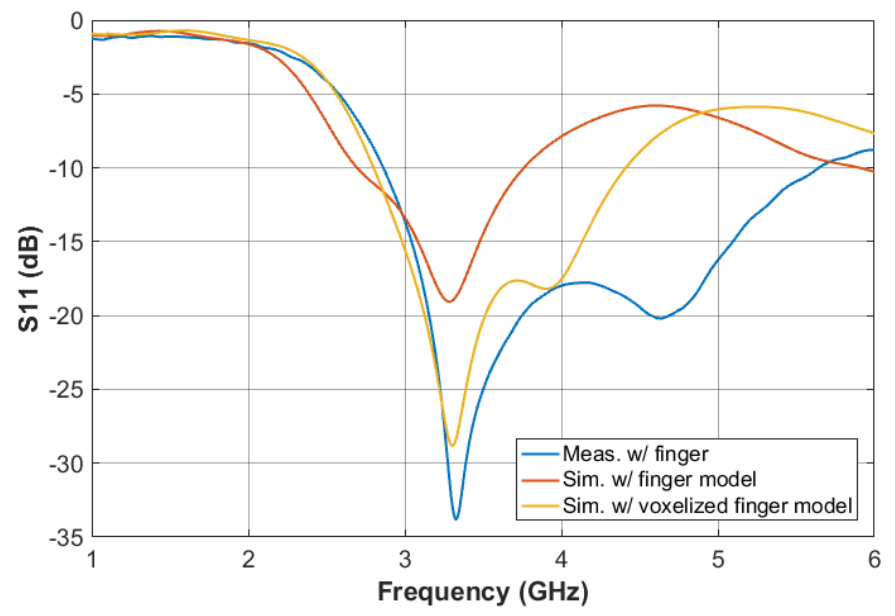

Fig. 13. Simulated frequency response of the sensor with the original and voxelized models and measured response with a finger

As it can be seen from Fig. 13, a better agreement between the voxelized model and real measurement was achieved with the new model compared to the original model.

After this, the thickness of the voxelized layer was varied from $4.8 \mathrm{~mm}$ down to $1.6 \mathrm{~mm}$ mimicking the compression of the fingertip due to applied pressure to observe its effects. The results are shown in Fig. 14. 


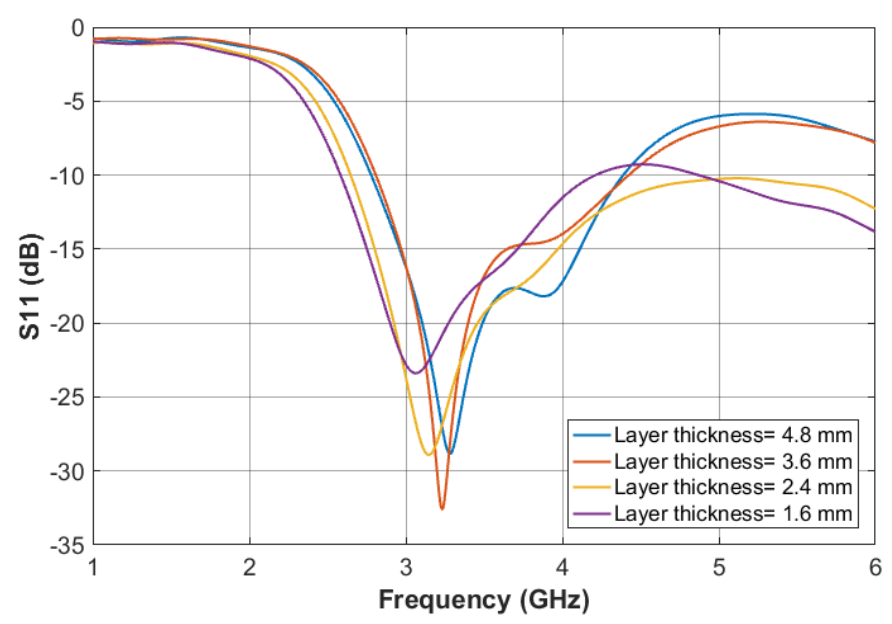

Fig. 14. Frequency response of the sensor with varied thickness of the voxelized layer

The resonance frequency decreases as the thickness decreases. About $90 \mathrm{MHz}$ downward shift was observed for $75 \%$ compression of the layer.

It can be concluded that the thickness of the mentioned biological layers plays a major role in achieving measurement repeatability. Layer thicknesses can be determined by measuring them and monitoring the pressure applied by the fingertip to ensure BGL measurement repeatability. A calibration procedure is vital to ensure repeatability of BGL measurements between different patients.

The authors are planning to manufacture synthetic fingertip phantoms to validate the concept by taking actual measurements and comparing with the computational models. Authors are also currently working on a novel system that will allow for accurate fingertip positioning on the sensor as well as measurement of layer thicknesses for BGL measurement calibration to be reported in future publications.

\section{PRESSURE DEPENDENCY OF BGL MEASUREMENTS}

To investigate the relationship between the resonance frequency and the pressure applied, a novel sensitive capacitive pressure measuring circuit that was reported in detail in [21] was used. The circuit utilizes a 24-bit Capacitance to Digital Converter (CDC) chip to monitor the capacitance of the RF sensor where the capacitance depends on the coupling between the sensor and the fingertip. The coupling increases as more pressure is applied. The circuit acts as an interface between the RF glucose sensor and the VNA utilizing the RF glucose sensor as a capacitive pressure sensor. The CDC uses a $16 \mathrm{kHz}$ excitation signal and the RF sensor provides a good sensitivity to proximity and pressure due to its highly capacitive nature by design. The circuit allows for continuous pressure and RF measurement. The circuit topology deployed is shown in Fig. 15. A high-pass filter (HPF) was used on the VNA line to block the excitation signal in that branch and an RF choke (RFC) on the CDC line to block the RF signal in that branch.

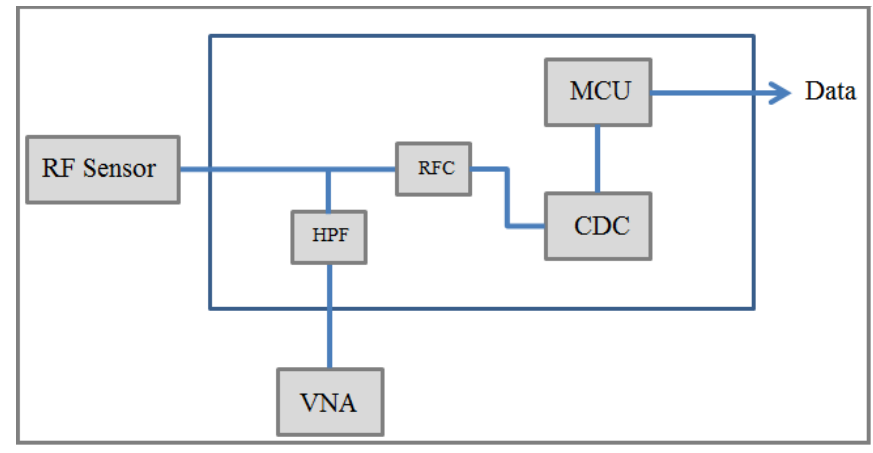

Fig. 15. Circuit topology of the proposed pressure sensing circuit

The pressure data is continuously sent to the computer by the microcontroller unit (MCU). The relationship between the resonance frequency and the pressure applied was established by continuously taking about 300 measurements in 3 minutes while varying the pressure applied onto the RF sensor. Pressure was varied randomly between light and firm levels and results were plotted in real-time in MATLAB [27] with the frequency response gathered from the VNA and the pressure data received from the proposed circuit. The experiment was repeated by taking data from 20 volunteering individuals. The obtained graph is shown in Fig. 16 for one of the data sets. The graph shows that the resonance frequency is almost a linear function of the pressure applied (in Arbitrary Units (AU)) and a good repeatability can be achieved for a specific pressure level.

The resonance frequency differs from person to person due to differences in the finger structure; however, the almost-linear relationship between the pressure applied and the resonance frequency is clearly observed for each individual.

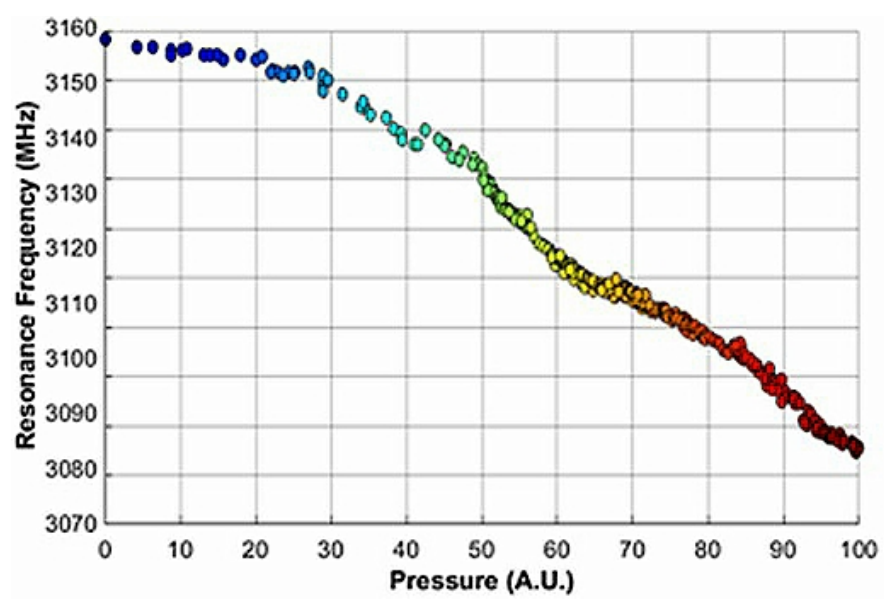

Fig. 16. Resonance frequency vs. pressure applied

The photograph of the pressure sensing circuit as well as the RF sensor in a custom 3D printed enclosure is shown in Fig. 17. 


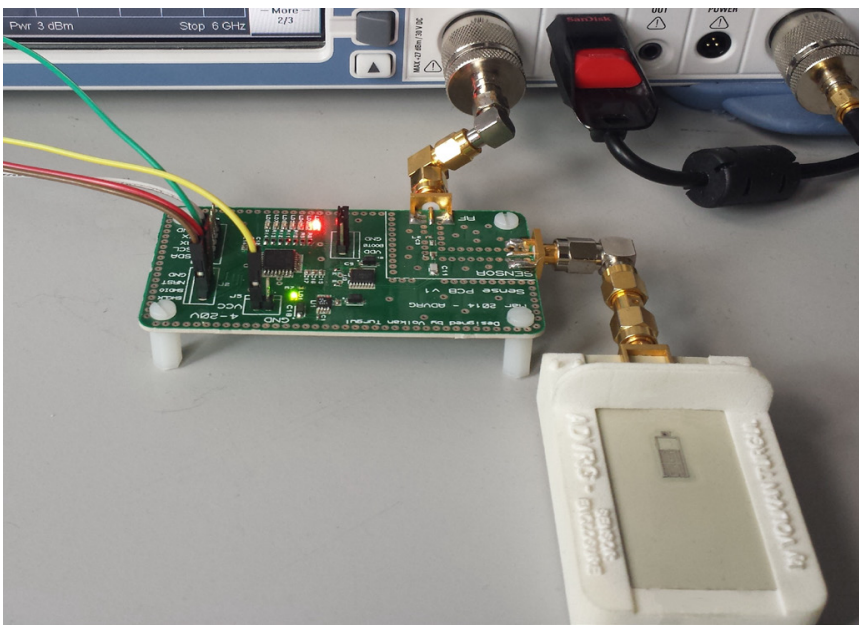

Fig. 17. Photograph of the pressure sensing circuit with the glucose sensor

Several different sensor enclosures were $3 \mathrm{D}$ printed as shown in Fig. 18 to accommodate for different finger types while ensuring correct finger positioning.

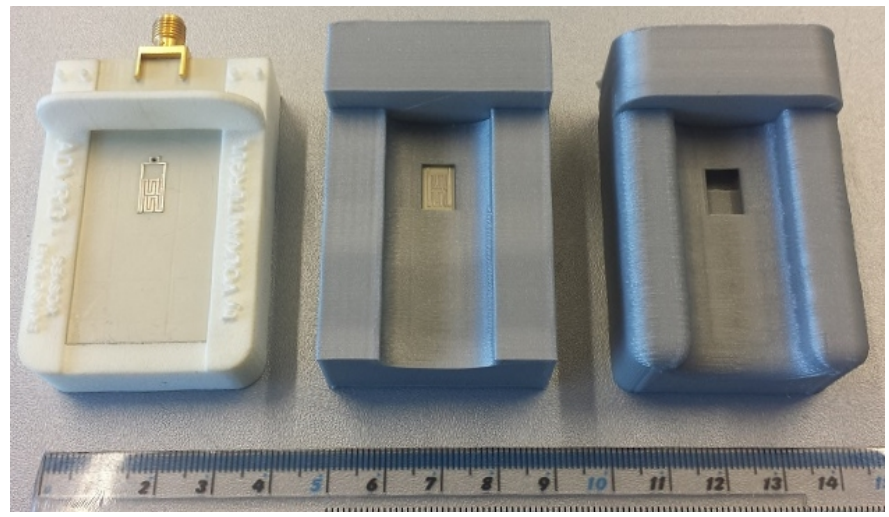

Fig. 18. Various sensor enclosures for measurements from fingertips

\section{DISCUSSION}

The parameters affecting the measurement accuracy are summarized in this section. It should be noted that these results are simulation based except the pressure measurements. It is expected that these results would draw attention to the challenges that could be faced when taking RF BGL measurements in terms of measurement accuracy and repeatability, which are key factors in BGL measurements. As mentioned in the previous section, the authors are working on ways to perform realistic measurements to quantify the denoted parameters and compare with the simulation results. Although these simulations were performed for the case of fingertips, the measurement challenges apply for any part of the body where the RF BGL measurement is taken from. The effects of the mentioned parameters are summarized in Table II.
TABLE II

SUMMARY OF PARAMETERS AND THEIR EFFECTS

\begin{tabular}{|l|l|}
\hline \multicolumn{1}{|c|}{ Parameter } & \multicolumn{1}{c|}{ Effect (Frequency shift) } \\
\hline Fingerprint variation & Up to $100 \mathrm{MHz}$ \\
\hline Biological layer thickness & Up to $90 \mathrm{MHz}$ \\
\hline Finger position & Up to $250 \mathrm{MHz}$ \\
\hline Pressure applied & Up to $80 \mathrm{MHz}$ \\
\hline Contaminants & Up to $80 \mathrm{MHz}$ \\
\hline
\end{tabular}

As it can be seen from the Table II, any error source may cause a frequency shifts around $100 \mathrm{MHz}$ and this may be in either direction. Considering that a combination of these errors may play a role during measurements, which can make it quite challenging to get accurate results.

\section{CONCLUSION}

RF non-invasive BGL measurement by tracking the changes in the permittivity of blood is a promising but challenging research topic. This is mainly because in the realistic BGL range the permittivity change is very limited which translates to a frequency shift of less than $8 \mathrm{MHz}$ in the sensor's frequency response. It was shown in this paper that external factors also play a major role in the sensor's response. The variation in fingerprint patterns, valley depth and biological layer thicknesses in the fingertips may cause a frequency shift of up to $100 \mathrm{MHz}$. These variations result due to the layer compression from the amount of pressure applied onto the sensor. Additionally, these parameters differ in each patient as well. The applied pressure can be accurately tracked using a pressure sensing circuit as proposed and layer thicknesses can be measured for calibration purposes. Moreover, finger positioning may cause a shift as high as $250 \mathrm{MHz}$ whose effect can be greatly reduced by housing the sensor in a custom enclosure to ensure finger position repeatability. Although the importance of these parameters were only emphasized for fingertips, irregularities such as skin shape and thickness, hair and biological layers present at the measurement site need to be considered carefully for any body part. The findings reported here show the importance of several parameters that need to be considered carefully to minimize measurement error, maximize repeatability between measurements for a patient and also the necessity of a calibration procedure to minimize measurement variations between different patients. To the authors' best knowledge these factors were not previously addressed in the open literature. The authors are currently investigating hybrid calibration procedures to mitigate the effects of all the mentioned error sources. 


\section{REFERENCES}

[1] "Diabetes Atlas, 6th ed.," International Diabetes Federation (IDF), 2014. [Online]. [Accessed 05 2016].

[2] R. Holt, C. Cockram, A. Flyvbjerg and B. Goldstein, Textbook of Diabetes, New York, NY: John Wiley \& Sons, 2011.

[3] S. Vashist, "Continuous Glucose Monitoring Systems: A Review," Diagnostics, vol. 3, no. 4, pp. 385-412, 2013.

[4] C. E. F. Amaral and B. Wolf, "Current development in non-invasive glucose monitoring," Medical Engineering \& Physics, vol. 30, no. 5, pp. 541$549,2008$.

[5] C. So, K. Choi, T. K. Wong and J. W. Chung, "Recent advances in noninvasive glucose monitoring,", vol. 5, pp. 45-52, 2012.," Medical Devices: Evidence and Research, vol. 5, pp. 45-52, 2012.

[6] V. V. Tuchin, Handbook Of Optical Sensing Of Glucose In Biological Fluids And Tissues, Boca Raton: CRC Press, 2009.

[7] G5 Mobile Continuous Glucose Monitoring (CGM) System, Dexcom Inc., 2017.

[8] Eversense CGM System, Senseonics Inc., 2017.

[9] J. Venkataraman and B. Freer, "Feasibility of non-invasive blood glucose monitoring: In-vitro measurements and phantom models," in International Symposium on Antennas and Propagation (APSURSI), Washigton, 2011.

[10] M. Hofmann, G. Fischer, R. Weigel and D. Kissinger, "MicrowaveBased Noninvasive Concentration Measurements for Biomedical Applications," IEEE Transactions on Microwave Theory and Techniques, vol. 61, no. 5, pp. 2195-2204, 2013.

[11] T. Karacolak, E. C. Moreland and E. Topsakal, "Cole-cole model for glucose-dependent dielectric properties of blood plasma for continuous glucose monitoring," Microwave and Optical Technology Letters, vol. 55, no. 5, pp. 1160-1164, 2013.

[12] K. K. Adhikari and N. Y. Kim, "Ultrahigh-Sensitivity Mediator-Free Biosensor Based on a Microfabricated Microwave Resonator for the Detection of Micromolar Glucose Concentrations," IEEE Transactions on Microwave Theory and Techniques, vol. 64, no. 1, pp. 319-327, 2016.

[13] U. Schwerthoeffer, R. Weigel and D. Kissinger, "A highly sensitive glucose biosensor based on a microstrip ring resonator," in Microwave Workshop Series on RF and Wireless Technologies for Biomedical and Healthcare Applications (IMWS-BIO), 2013.

[14] T. Yilmaz, R. Foster and Y. Hao, "Towards Accurate Dielectric Property Retrieval of Biological Tissues for Blood Glucose Monitoring,"

IEEE Transactions on Microwave Theory and Techniques, vol. 62, no. 12, pp. 3193-3204, 2014.

[15] I. Gouzouasis, H. Cano-Garcia, I. Sotiriou, S. Saha, G. Palikaras, P. Kosmas and E. Kallos1, "Detection of Varying Glucose Concentrations in Water Solutions Using a Prototype Biomedical Device for Millimeter-Wave Non-invasive Glucose Sensing," in 10th European Conference on Antennas and Propagation (EuCAP), Davos, 2016.

[16] "Gluco Track," Integrity Applications Inc., Israel, 2017.

[17] "GlucoWise," MediWise Ltd., U.K., 2017.

[18] B. Jean, E. Green and M. McClung, "A microwave frequency sensor for non-invasive blood-glucose measurement," in IEEE Sensors Applications Symposium, 2008

[19] T. Yilmaz, R. Foster and Y. Hao, "Patch resonator for non-invasive detection of dielectric property changes in biological tissues," in Antennas and Propagation Society International Symposium (APSURSI), 2012.

[20] V. Turgul and I. Kale, "Characterization of the Complex Permittivity of Glucose/Water Solutions for Non-invasive RF/Microwave Blood Glucose Sensing," in IEEE International Instrumentation and Measurement Technology Conference (I2MTC), Taipei, 2016.

[21] V. Turgul and I. Kale, "A Novel Pressure Sensing Circuit for Noninvasive RF/Microwave Blood Glucose Sensors," in IEEE Mediterranean Microwave Symposium (MMS), Abu Dhabi, 2016.

[22] V. Turgul and I. Kale, "Influence of fingerprints and finger positioning on accuracy of RF blood glucose measurement from fingertips," IET Electronics Letters, vol. 53, no. 4, pp. 218-220, 2016.

[23] "CST Microwave Studio," Computer Simulation Technology AG, Darmstadt, 2015.

[24] "ISO 15197:2013, In vitro diagnostic test systems - Requirements for blood-glucose monitoring systems for self-testing in managing diabetes mellitus," International Organization for Standardization, 2013. [Online]. [Accessed 06 2017].

[25] S. Back, Y. Lee, S. Lee and G. Son, "Moisture-insensitive optical fingerprint scanner based on polarization resolved in-finger scattered light," Optic Express 24, pp. 19195-19202, 2016.
[26] S. Richmond, "Do fingerprint ridges and characteristics within ridges change with pressure?," Australian Federal Police Forensic Services, 2014.

[27] “MATLAB R2015a," The MathWorks Inc, Massachusetts, 2015.

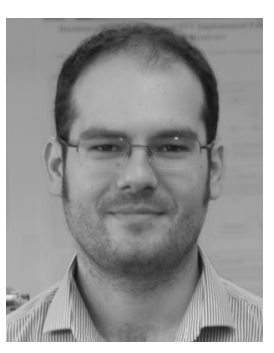

Volkan Turgul (S'14) received the B.Sc. degree in electronic engineering from the Middle East Technical University, Northern Cyprus Campus, Turkey in 2010, the M.Sc. degree in mobile, wireless and broadband communications from the University of Westminster, London, U.K. in 2011 and the Ph.D. degree at the University of Westminster, London, U.K in 2017. He is currently doing post-doctoral research at the University of Westminster working on novel non-invasive blood glucose measurement and biomedical diagnostic systems.

His research interests include instrumentation and measurement, miniaturized microstrip RF structures, noninvasive sensors and digital signal processing.

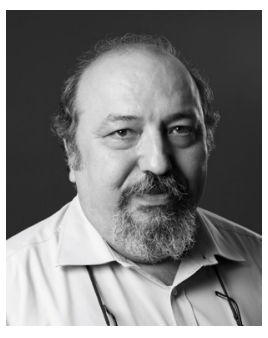

Izzet Kale (M'88) was born in Cyprus. $\mathrm{He}$ received the B.Sc. (honors) degree in electrical and electronic engineering from the Polytechnic of Central London, London, U.K., the M.Sc. degree in the design and manufacture of microelectronic systems from Edinburgh University, Edinburgh, U.K., and the $\mathrm{Ph} . \mathrm{D}$. degree in techniques for reducing digital filter complexity from the University of Westminster, London.

He joined the staff with the University of Westminster in 1984 and he has been with them since and currently the Head of the Department of Engineering. He is the founder and the Director of the Applied Digital Signal Processing (DSP) and Very Large Scale Integration System (VLSI) Research Group, the University of Westminster, where he has undertaken and lead numerous applied research and development projects and contracts for European, U.S., and Japanese corporations, working on innovative silicon integrated product development for commercial applications. He is currently a Professor of applied DSP and VLSI systems. His research and teaching activities include digital and analog signal processing, silicon circuit and system design, digital filter design and implementation, and analog/digital and digital/analog sigmadelta converters. He is currently working on efficiently implementable ultralow-power DSP algorithms/architectures and sigma-delta modulator structures for use in the communications and biomedical industries as well as invasive and non-invasive biomedical sensors and systems. 